\title{
Prediction of Chaos in Electrostatically Actuated Arch Micro-Nano Resonators: Analytical Approach
}

\author{
Farid Tajaddodianfar ${ }^{1}$, Hossein Nejat Pishkenari $^{2}$, Mohammad Reza Hairi Yazdi ${ }^{*}$ \\ ${ }^{1}$ School of Mechanical Engineering, College of Engineering, University of Tehran, Tehran-Iran, \\ tajaddodian@ut.ac.ir \\ ${ }^{2}$ Center of Excellence in Design, Robotics and Automation, School of Mechanical Engineering, \\ Sharif University of Technology, Tehran - Iran, nejat@ sharif.edu \\ $3^{3 *}$ Corresponding Author, School of Mechanical Engineering, College of Engineering, University \\ of Tehran, Tehran - Iran, myazdi@ut.ac.ir , Tel.: +98 2188337123
}

\begin{abstract}
Chaotic vibrations of a bistable resonator comprised of doubly clamped shallow arch under simultaneous harmonic AC and static DC distributed electrostatic actuation are investigated. A single degree of freedom model obtained by application of the Galerkin decomposition method to the Euler-Bernoulli shallow arch equation is used for the studies. The bistable arch possessing an asymmetric double-well potential is vulnerable to homoclinic chaos at certain values of parameters. We have implemented the Melnikov's method to derive a necessary condition for the initiation of chaos in this type of resonators. Moreover, we have used a heuristic chaos prediction criterion, together with an analytical solution for the arch's frequency response given by the multiple scales method, to derive an analytical expression for prediction of chaos in the forced vibrations of the arch micro/nano-resonators. Numerical simulations confirm validity of the obtained analytical expressions.
\end{abstract}

Keywords: MEMS/NEMS; Arch resonator; Chaos; Melnikov's method; Multiple Scales Method

\section{Introduction}

Along with the rapid development of Micro- and Nano-Electro-Mechanical-Systems (M/NEMS), a growing attention has been paid to the bistable M/NEMS devices capable of operating in more than one stable configuration at fixed values of all actuation parameters. This growing attention is motivated by specific applications including MEMS based memories [1], bandpass filters [2], switches [3], microrelays [4], microvalves [5] and etc. Such a bistable device is possible to be constructed by a doubly clamped initially curved microbeam having two convex and concave stable configurations; the curviness of which is possible to be achieved either by micromachining or buckling of a straight beam under compressive axial load [6,7]. Structural instability, known as snap-through buckling, is responsible for precipitate transition of the arch from convex to concave configuration and vice versa. Various actuation mechanisms, including electro-thermal 
[8], electromagnetic [9], light pressure [10], mechanical touch [11] and electrostatic [12], have been reported for triggering this precipitate motion.

Electrostatically actuated M/NEMS devices are mostly vulnerable to well-known structural instability, known as pull-in, which addresses the sudden collapse of the structure due to inherent softening effect of the electrostatic force. During the past decades, lots of works, recently reviewed by Zhang et al. [13], have been reported focusing on the investigation and characterization of pull-in either as a desirable phenomenon, such as in RF MEMS switches [14], or as an undesirable phenomenon, e.g. in capacitive MEMS [15] applications. The pull-in instability, as well as the snap-through, is characterized by state of the system prior to the instability: i) the static pull-in which refers to the pull-in occurring under quasi-static loading [16], and ii) the dynamic pull-in which addresses the pull-in instability in the oscillatory systems [17].

Static pull-in and snap-through instabilities in electrostatically actuated arch MEMS devices have been widely addressed in the literature. Krylov et al. [12,18] used both theoretical and experimental approaches for the investigation of pull-in and snap-through instabilities in these bistable MEMS devices. Das and Batra [19] implemented the finite and boundary element methods to study the snap-through and pull-in instabilities. Zhang et al. [20] suggested making use of snap-through motion in the design of highly sensitive low-power sensors.

Few researches have been directed toward the resonance behaviors and nonlinear vibrations of electrostatically actuated arch resonators. The dynamic snap-through instability prompted by mechanical resonance is studied by Casals-Terre et al [21]. Ouakad and Younis [7], studied an arch resonator actuated by harmonic electrostatic force. Younis et al. [6] investigated the arch resonator both experimentally and theoretically. They suggested application of the electrostatically actuated arch resonator as bandpass filters, and recently have reported further discussions focusing on this type of application [2,22]. Also, recently Tajaddodianfar et al. [23], have reported detailed discussions on the bistable nature of vibrations in the electrostatically actuated arch resonators using analytical solutions.

Regarding various nonlinearities, MEMS-NEMS devices are vulnerable to chaos in their working regimes. Chaotic vibrations can be desirable in specific applications such as very sensitive sensors. Seleim et al. [24] investigated chaos in a closed loop cantilever MEMS resonator, suggesting application of chaotic resonator for construction of highly sensitive sensors. Also, Yin and Epureanu [25] implemented chaotic resonators for sensing applications. In most of the applications, chaos is interpreted as an undesired phenomenon deteriorating the system performance. Thus, many researchers aim to study the chaotic regimes of the resonator and avoid chaos in practice. Suppression of chaos using a control strategy is reported by Park et al. [26]. Mayoof and Hawwa [27] studied chaos in harmonically excited curved carbon nanotube using numerical simulations. The maximal Lyapunov exponent criterion is used by Aghababa [28] for chaos detection in fractional-order MEMS resonators. Amorim et al. [29] investigated 
chaotic behaviors of a doubly clamped beam resonator with various nonlinear effects using a single degree of freedom model. Determination of chaotic regions in the system's parameter space using analytical approaches provides a useful rapid tool for chaos prediction and prevention in practice. The Melnikov method is a popular technique in the literature for determination of the system parameters leading to chaos [30-32]. Nayfeh et al. [33] numerically evaluated the Melnikov function in a resonant gas sensor in order predict a critical value for the amplitude of the harmonic electrostatic load. Nejat Pishkenari et al. [34] proposed a heuristic method for prediction of chaos in atomic force microscope possessing multi-well potential function. Recently, Maani et al. [35] have presented a chaos prediction algorithm for MEMSNEMS resonators with symmetric double-well potential function. Later, they reported further discussions in the study of chaos in MEMS-NEMS resonators [36].

Although significant progress has been made so far in the study of nonlinear dynamics of electrostatically actuated bistable arch resonators, some main issues are still not well-discussed. Among them is the possibility of chaos in this type of arch resonators. However, to the authors' best knowledge, analytical solutions for the chaos detection in this type of bistable M/NEMS resonators have not been proposed. In this paper, we have investigated chaotic vibrations of a bistable MEMS resonator comprised of a double clamped initially curved microbeam actuated by simultaneous harmonic AC and static DC distributed electrostatic force. A single-degree-offreedom equation of motion, obtained by application of single-mode Galerkin decomposition method to the governing Euler-Bernoulli shallow arch equation with mid-plane stretching effects and distributed electrostatic force, is used for investigations in this paper. Primarily, based on the shape of the potential energy function, the possible arch configurations are classified into three categories which one of them presents asymmetric double-well potential with the possibility of undergoing chaos. An analytical approach, the multiple scales method, is used to describe the nonlinear oscillatory behavior of the arch near its first fundamental frequency. We have analytically evaluated the Melnikov's function to derive a closed form relation for prediction of chaos in the parameter space of the resonator. Moreover, using the analytical frequency response solution given by the multiple scales method together with a chaos prediction algorithm, named the maximum velocity criterion proposed by Nejat Pishkenari et al. [34], we have derived an analytical formula for prediction of steady state chaos in the nonlinear forced vibrations of the arch resonator with asymmetric double-well potential. We have conducted various numerical simulations to validate the obtained analytical expressions.

The rest of the paper is organized as follows. Section 2 describes the mathematical model and the corresponding potential energy function. Section 3 presents the analytical frequency response solution obtained by the multiple scales method. In Section 4, we have described the proposed approach to derive Melnikov's analytical formula for chaos detection. We have also applied the maximum velocity criterion to derive analytical expression for chaos prediction. In Section 5, we have validated the obtained solutions by numerical simulations, and have evaluated effectiveness 
of the obtained expressions for description of chaotic regions in parameter space of the arch resonator. Further discussions and conclusions are presented in Section 6.

\section{Mathematical Modeling}

\subsection{Reduced order model}

Consider a doubly clamped arch shaped microbeam of length $L$, width $b$, thickness $d$ under combined harmonic AC and static DC distributed electrostatic actuation, as shown in Figure 1. Supposing that the initial curvature of the beam is given by $w_{0}(x)$ with $h_{0}=\left|w_{0}(L / 2)\right|$, the arch's displacement under the distributed electrostatic load is given by $w(x, t)$ measured from the initial configuration in the positive direction of the $z$ axis.

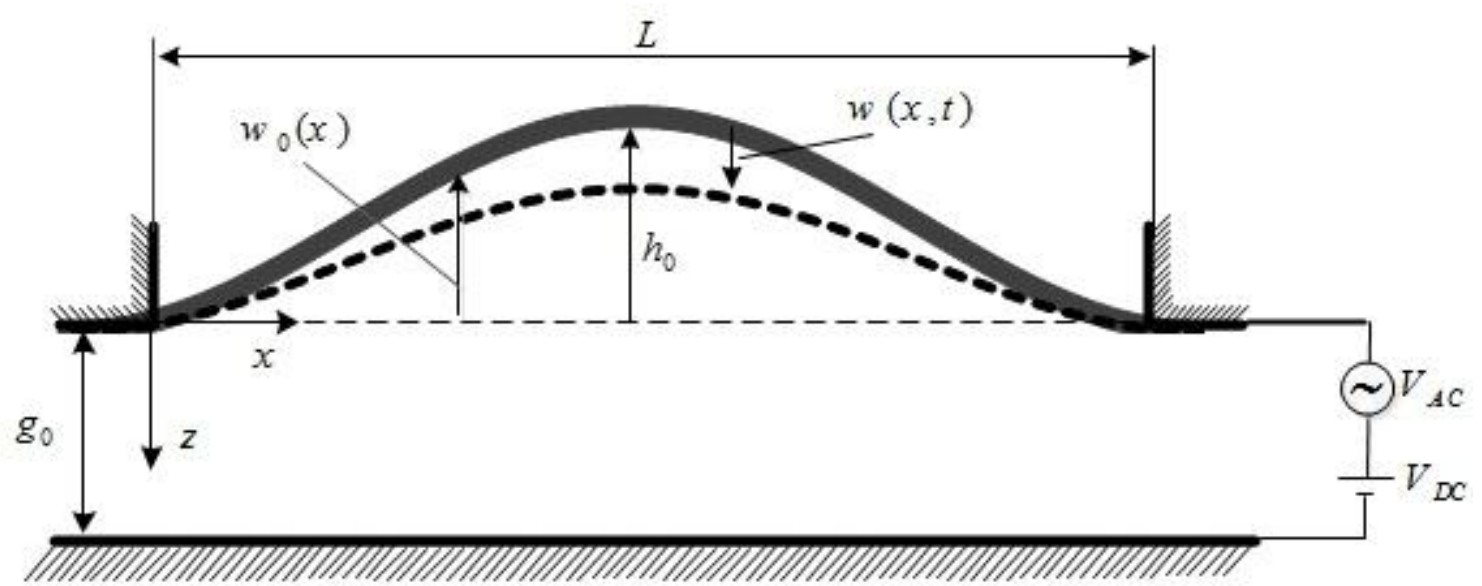

Figure 1: Arch resonator under harmonic electrostatic actuation

Using the given coordinates and parameters, the equation of motion of the shallow arch in the framework of Euler-Bernoulli beam assumptions is obtained [6]:

$$
\begin{aligned}
& \rho A \frac{\partial^{2} w}{\partial t^{2}}+C_{v} \frac{\partial w}{\partial t}+\tilde{E} I_{y} \frac{\partial^{4} w}{\partial x^{4}}-\frac{\tilde{E} A}{2 L}\left[\int_{0}^{L}\left(\left(\frac{\partial w}{\partial x}\right)^{2}+2 \frac{\partial w}{\partial x} \frac{d w_{0}}{d x}\right) d x\right]\left(\frac{\partial^{2} w}{\partial x^{2}}+\frac{d^{2} w_{0}}{d x^{2}}\right) \\
& =\frac{\varepsilon_{0} b\left(V_{D C}+V_{A C} \cos \left(\Omega_{0} t\right)\right)^{2}}{2\left(g_{0}-w_{0}-w\right)^{2}}
\end{aligned}
$$

where $A$ is the cross-sectional area and $C_{v}$ is the viscous damping coefficient; while $E^{t}, I_{y}, r$ and $\mathrm{W}_{0}$ represent the effective Young's modulus, area moment of inertia, mass density and the harmonic load frequency, respectively. Also, $e_{0}=8.854^{\prime} 10^{-12} \mathrm{~F} / \mathrm{m}$ is the vacuum permittivity. Boundary conditions associated with equation (1) are given as: 
$w(0, t)=w(L, t)=0$,

$\left.\frac{\prod w}{\llbracket x}\right|_{x=0}=\left.\frac{\prod w}{\llbracket x}\right|_{x=L}=0$

We further suppose that the amplitude of the harmonic load is small compared to that of the DC static load, imposing a small value for the ratio $R=V_{A C} / V_{D C}$. Equation (1) is converted to the following dimensionless form using the non-dimensional parameters given in Table 1.

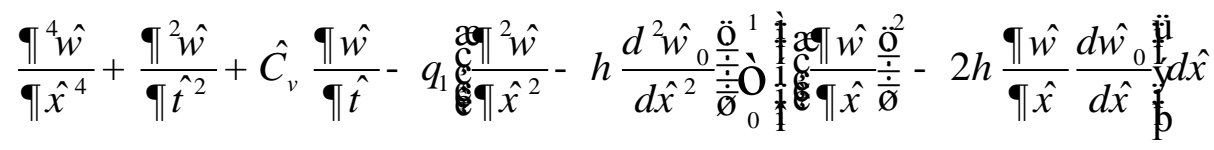

$=\frac{b\left(1+2 R \cos \left(\hat{w}_{0} \hat{t}\right)\right)}{\left(1+h \hat{w}_{0}-\hat{w}\right)^{2}}$

with the boundary conditions:

$\hat{w}(0, \hat{t})=\hat{w}(1, \hat{t})=0$,

$\left.\frac{\llbracket \hat{w}}{\llbracket x}\right|_{\hat{x}=0}=\left.\frac{\llbracket \hat{w}}{\llbracket \hat{x}}\right|_{\hat{x}=1}=0$

Note that, based on the coordinate system shown in Figure 1, the initial curvature function $w_{0}(\mathrm{x})$ is replaced by $-h_{0} \hat{w}_{0}(\hat{\mathrm{x}})$; where $\hat{w}_{0}(\hat{\mathrm{x}})$ is a dimensionless function with the property $\hat{w}_{0}(1 / 2)=1$. The dimensionless quantity $q_{1}$, called the stretching parameter, reflects the midplane stretching nonlinear effects; while the dimensionless DC voltage parameter $b$ is associated with nonlinear electrostatic force.

Table 1: Dimensionless parameters

\begin{tabular}{|l|l|l|l|l|}
\hline$\hat{x}=\frac{x}{L}$, & $\hat{b}=\frac{b}{g_{0}}$, & $\hat{d}=\frac{d}{g_{0}}$, & $b=\frac{e_{0} b L^{4} V_{D C}^{2}}{2 g_{0}^{3} E P_{y}}$, & $\hat{w}_{0}=\frac{\mathrm{W}_{0} t}{\hat{t}}$, \\
\hline$\hat{w}=\frac{w}{g_{0}}$, & $h=\frac{h_{0}}{g_{0}}$, & $q_{1}=\frac{b d g_{0}^{2}}{2 I_{y}}$, & $\hat{t}=t \sqrt{\frac{E^{\theta} P_{y}}{r b d L^{4}}}$, & $\hat{C_{v}}=\frac{C_{v} L^{2}}{\sqrt{E^{\theta} \varphi_{y} b d r}}$, \\
\hline
\end{tabular}

For derivation of a single-degree-of-freedom model using the Galerkin projection method, the arch's deflection is supposed to be approximated by its first mode shape:

$$
\hat{w}(\hat{x}, \hat{t}) ; q(\hat{t}) j(\hat{x})
$$

where $j(\hat{x})$ represents the first normalized mode shape of a clamped-clamped straight beam [12] which satisfies boundary conditions (4), as: 


$$
\begin{aligned}
& j(\hat{x})=\cosh \left(l_{1} \hat{x}\right)-\cos \left(l_{1} \hat{x}\right)-\frac{\cosh \left(l_{1}\right)-\cos \left(l_{1}\right)}{\sinh \left(l_{1}\right)-\sin \left(l_{1}\right)}\left[\sinh \left(l_{1} \hat{x}\right)-\sin \left(l_{1} \hat{x}\right)\right], \quad l_{1}=4.7300, \\
& j(\hat{x})=\frac{J(\hat{x})}{\max _{\hat{x} \hat{1}[0,1]}(j(\hat{x}))},
\end{aligned}
$$

Substituting from (5) into (3), multiplying both sides by $j(\hat{x})$, integrating over the arch length and following a straight-forward mathematical procedure, the following reduced-order equation is derived:

$\frac{d^{2} q}{d \tau^{2}}+\mu \frac{d q}{d \tau}+\left(1+2 h^{2} \alpha_{1}\right) q-3 \alpha_{1} h q^{2}+\alpha_{1} q^{3}-\frac{\beta\left(1+2 R \cos \left(\omega_{0} \tau\right)\right)}{b_{11}} \int_{0}^{1} \frac{\varphi(\hat{x})}{[1+(h-q) \varphi(\hat{x})]^{2}} d \hat{x}=0$

where $t=\hat{t} \sqrt{b_{11} / m_{11}}, m=\hat{C}_{v} \sqrt{m_{11} / b_{11}} \quad, a_{1}=q_{1} s_{11}^{2} / b_{11}$ and $w_{0}=\hat{w}_{0} \sqrt{m_{11} / b_{11}}$ with the following definitions for the constants $m_{11}, b_{11}$ and $s_{11}$ :

$$
\begin{aligned}
& m_{11}=\underset{0}{\mathbf{O}} j^{2}(\hat{x}) d \hat{x} \\
& b_{11}=\underset{0}{1} \grave{\mathbf{O}}_{0}\left(\frac{d^{2} j}{d \hat{x}^{2}}\right)^{2} \mathrm{~d} \hat{\mathrm{x}} \\
& s_{11}=\underset{\mathrm{O}}{\mathbf{\mathrm { j }}}\left(\frac{d j}{d \hat{x}}\right)^{2} d \hat{x}
\end{aligned}
$$

An analytical solution for the integral function arising in equation (7) is not available using the mode shape given by equation (6). However, one can obtain an analytical approximation for the modal electrostatic force, provided that $j(\hat{\mathrm{x}})$ is replaced by the approximation $(1-\cos (2 p \hat{\mathrm{x}})) / 2$ , which yields [18]:

$$
\mathrm{Ò̀}_{0}^{1} \frac{j(\hat{x})}{[1+(h-q) j(\hat{x})]^{2}} d \hat{x}=\frac{1}{2 \sqrt{(1+h-q)^{3}}}
$$

Thus, using (11) one can re-write equation (7) in the following form as the reduced order single degree of freedom nondimensionalized equation of motion [23], which will be used in the rest of this paper for our investigations:

$$
\begin{aligned}
& \frac{d^{2} q}{d \tau^{2}}+\mu \frac{d q}{d \tau}+F(q)=0 \\
& F(q)=\left(1+2 h^{2} \alpha_{1}\right) q-3 \alpha_{1} h q^{2}+\alpha_{1} q^{3}-\frac{\beta\left(1+2 R \cos \left(\omega_{0} \tau\right)\right)}{2 b_{11} \sqrt{(1+h-q)^{3}}}
\end{aligned}
$$




\subsection{Potential Energy and Bistability}

To derive potential energy function, we first eliminate the AC loading parameter due to its small value. Then, the potential function $U(q)$ can easily be calculated by integrating $F(q)$ such as:

$U(q)=\grave{\mathrm{o}} F(q) d q=\frac{1}{2}\left(1+2 h^{2} a_{1}\right) q^{2}-a_{1} h q^{3}+\frac{1}{4} a_{1} q^{4}-\frac{b}{b_{11} \sqrt{1+h-q}}$

It is clear that the shape of the potential function depends on $q$ and consequently on $b$ and $h$. Moreover, extremums of $U(q)$ are equal to equilibrium or fixed points of equation (12). Thus, for various values of $b$ and $h$, various numbers of minima and maxima appear in the potential function. For certain values of $h>h^{*}$, for which bistability occurs [18], different shapes of $U(q)$ are possible depending on the applied DC voltage denoted by parameter $b$. We can sort the possible potential function shapes into three major categories in Table 2. Note that both $q_{01}$ and $q_{02}$ stand for the stable fixed points, where $q_{s}$ and $q_{p}$ represent unstable fixed points relating to the snap-through and the pull-in instabilities, respectively.

Table 2 clarifies that only for special values of $h$ and $b$ the arch is bistable. However, in the bistability region, the system presents a mainly-asymmetric double-well potential function; thus it can be vulnerable to nonlinear behaviors including snap-through and chaos [23].

Table 2. Various categories based on the shape of potential function

\begin{tabular}{|c|c|c|c|}
\hline Label & Description & Potential Function & Phase Plane \\
\hline $\begin{array}{c}\text { Category } \\
\text { A }\end{array}$ & $U\left(q_{01}\right)<U\left(q_{p}\right)$ & & \\
\hline $\begin{array}{c}\text { Category } \\
\text { B }\end{array}$ & $\begin{array}{c}U\left(q_{01}\right)<U\left(q_{02}\right)<U\left(q_{s}\right)<U\left(q_{p}\right) \\
\text { Or } \\
U\left(q_{01}\right) ; U\left(q_{02}\right)<U\left(q_{s}\right)<U\left(q_{p}\right) \\
\text { Or } \\
U\left(q_{02}\right)<U\left(q_{01}\right)<U\left(q_{s}\right)<U\left(q_{p}\right)\end{array}$ & & 4 \\
\hline $\begin{array}{c}\text { Category } \\
\text { C }\end{array}$ & $U\left(q_{p}\right)<U\left(q_{s}\right)$ & & \\
\hline
\end{tabular}




\subsection{Vibrations in the vicinity of equilibrium states}

We suppose that at first the static DC load is applied; and the arch comes to rest in an equilibrium configuration. Then, vibrations in the vicinity of the equilibrium state are triggered by application of small amplitude harmonic AC load. In order to account for this assumption, we introduce the following change of variables:

$q(t)=q_{0}+u(t)$

where $q_{0}$ stands for the equilibrium configuration under the applied DC voltage. Substituting equation (14) into equation (12) and expanding nonlinear terms in powers of $u$ yields the governing equation of motion in terms of $u(t)$ as below [23]:

$\frac{d^{2} u}{d t^{2}}+m \frac{d u}{d t}+m_{1} u+m_{2} u^{2}+m_{3} u^{3}+\left(s_{0}+s_{1} u+s_{2} u^{2}+s_{3} u^{3}\right) R \cos \left(w_{0} t\right)=0$

Note that in the derivation of equation (15), the nonlinear electrostatic term in equation (12) is approximated up to the $3^{\text {rd }}$ order using Taylor's expansion method which is sufficiently accurate for our next calculations. Also, coefficients given in equation (15) are obtained using basic calculations, as followings [23]:

$$
\begin{aligned}
& m_{1}=1+2 h^{2} a_{1}-6 h a_{1} q_{0}+3 a_{1} q_{0}^{2}-\frac{3 b}{4 b_{11}}\left(1+h-q_{0}\right)^{\frac{-5}{2}} \\
& m_{2}=-3 h a_{1}-3 q_{0} a_{1}-\frac{15 b}{16 b_{11}}\left(1+h-q_{0}\right)^{\frac{-7}{2}} \\
& m_{3}=a_{1}-\frac{35 b}{32 b_{11}}\left(1+h-q_{0}\right)^{\frac{-9}{2}} \\
& s_{0}=-\frac{b}{b_{11}}\left(1+h-q_{0}\right)^{\frac{-3}{2}} \\
& s_{1}=-\frac{3 b}{2 b_{11}}\left(1+h-q_{0}\right)^{\frac{-5}{2}} \\
& s_{2}=-\frac{15 b}{8 b_{11}}\left(1+h-q_{0}\right)^{\frac{-7}{2}} \\
& s_{3}=\frac{35 b}{16 b_{11}}\left(1+h-q_{0}\right)^{\frac{-9}{2}}
\end{aligned}
$$

Note that $q_{0}$ and $b$ satisfy the static fixed points equation $F(q)=0$ with $R=0$. Furthermore, we assume that the $\mathrm{AC}$ actuation frequency is close to the fundamental frequency of the arch:

$w_{0}=l+s$

where $s$ is a detuning parameter and $l=\sqrt{m_{1}}$ denotes the first fundamental frequency of the arch. 


\section{Analytical Frequency Response}

The multiple scales method (MSM) can be used to derive an analytical solution for the nonlinear vibrations in the vicinity of equilibrium points governed by equation (15). To this aim, supposing small amplitude of vibrations, and with $e$ as a small parameter, $u(t)$ in equation (15) can be replaced by $u(t)=e v(t)$ which yields:

$\frac{d^{2} v}{d t^{2}}+\hat{m} e \frac{d v}{d t}+m_{1} v+m_{2} e v^{2}+m_{3} e^{2} v^{3}+\hat{R} e\left(s_{0}+s_{1} e v+s_{2} e^{2} v^{2}+s_{3} e^{3} v^{3}\right) \cos \left(w_{0} t\right)=0$,

where $m=\hat{m} e$ and $R=\hat{R} e^{2}$ are further assumed due to the large quality factor and small AC amplitude assumptions, respectively. Moreover, $s=\hat{s} e^{2}$ is assumed which implies the actuation frequency to be close to the natural frequency of the resonator. Time scales are then defined as $T_{n}=e^{n} t, n=1,2,3$ which gives the following time derivative operators:

$\frac{d}{d t}=D_{0}+e D_{1}+e^{2} D_{2}$

$\frac{d^{2}}{d t^{2}}=D_{0}^{2}+2 e D_{0} D_{1}+e^{2} D_{1}^{2}+2 e^{2} D_{0} D_{2}$

where $D_{n}=d / d T_{n}, n=1,2,3$. The displacement $v(t)$ in equation (18) is asymptotically expanded with respect to $e$ :

$v(t ; e)=v_{0}\left(T_{0}, T_{1}, T_{2}\right)+e v_{1}\left(T_{0}, T_{1}, T_{2}\right)+e^{2} v_{2}\left(T_{0}, T_{1}, T_{2}\right)+O\left(e^{3}\right)$

Substituting (19) and (20) into (18), and equating the like powers of $e$ to zero, one finds the following ordinary differential equations:

$D_{0}^{2} v_{0}+l^{2} v_{0}=0$

$D_{0}^{2} v_{1}+l^{2} v_{1}+\hat{m} D_{0} v_{0}+2 D_{0} D_{1} v_{0}+m_{2} v_{0}^{2}+\hat{R} \cos \left(w_{0} t\right)=0$,

$D_{0}^{2} v_{2}+l^{2} v_{2}+\hat{m} D_{1} v_{0}+\left(D_{1}^{2}+2 D_{0} D_{2}\right) v_{0}+m_{3} v_{0}^{3}$

$+\hat{m} D_{0} v_{1}+2 D_{0} D_{1} v_{1}+2 m_{2} v_{0} v_{1}+\hat{R} s_{1} v_{0} \cos \left(w_{0} t\right)=0$

Equation (21) yields the following solution:

$v_{0}=Z_{0}\left(T_{1}, T_{2}\right) e^{i l T_{0}}+\bar{Z}_{0}\left(T_{1}, T_{2}\right) e^{-i l T_{0}}$

where $Z_{0}$ and $\bar{Z}_{0}$ are complex conjugates. Substituting (24) into (22) and eliminating secular terms yields:

$v_{1}=\frac{m_{2}}{3 l^{2}}\left(Z_{0}^{2} e^{2 i l T_{0}}+\bar{Z}_{0}^{2} e^{-2 i l T_{0}}+2 Z_{0} \bar{Z}_{0}\right)$

$\frac{\mathbb{\Phi} A_{0}}{\llbracket T_{1}}=-\frac{\hat{m}}{2} A_{0}-\frac{\hat{R}}{4 l} e^{i \hat{s}^{2} T_{0}}$ 
Substituting (24)-(26) into (23), and eliminating secular terms gives:

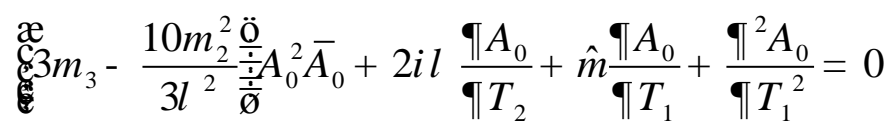

Representing $A_{0}$ in its polar form, and entering it into (25), (26) and (27), and straightforwardly manipulating the mathematical expressions, one finds the following expression as the frequency response equation corresponding to equation (18) (see [35,37] for more details):

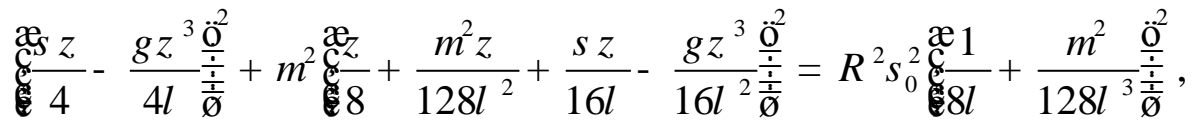

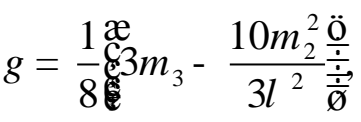

where $z$ is the amplitude of the vibration. With the all geometrical and actuation parameters known, the algebraic equation (28) is solved for the unknown $z$ representing the amplitude of $u(t)$. Figure 2 displays the frequency response of a typical arch resonator of dimensions $L=1000 \mathrm{~mm}, d=3 \mathrm{~mm}, b=30 \mathrm{~mm}$ and $g_{0}=10 \mathrm{~mm}$; which result in dimensionless constants $a_{1}=7.993$ and $b_{11}=198.462$. These parameters are used throughout this paper. Other parameters used for derivation of the frequency response curve given in Figure 2 are set as: $h=0.34, b=128, R=0.01$ and $m=0.03$ with the arch being at rest initially at its lower equilibrium configuration.

Figure 2 also verifies accuracy of the given analytical solution by comparing it with the results of the numerical simulations. In order to obtain the numerical results, the arch resonator governed by equation (12) which is actuated with a frequency lower than the fundamental frequency ( $s<0$ ) is simulated, and the amplitude of the steady state response is recorded. Then, the actuation frequency is increased, and the simulation is repeated for the new frequency; while at each step the final state of the system is used as the initial condition of the next step. This constitutes the forward frequency sweep path in Figure 2. The backward sweep path is formed by decreasing the actuation frequency. Note that Figure 2 confirms validity of the analytical MSM frequency response (28) for the analysis of the nonlinear dynamic model (12) without any further approximation.

Figure 2 confirms that the amplitude of the response predicted by the stable branch of the MSM solution agrees well with the amplitude of the steady state numerical simulations both in forward and backward frequency sweeps. The middle branch of the MSM solution predicts unstable solutions which are not reached out by the explained simulation procedure. For the given value of the parameters, the arch acts as a typical resonator with softening type frequency response. For other parameter values, the arch can present hardening type frequency responses, or bistable vibrations with consequent dynamic snap-through switching [23]. 


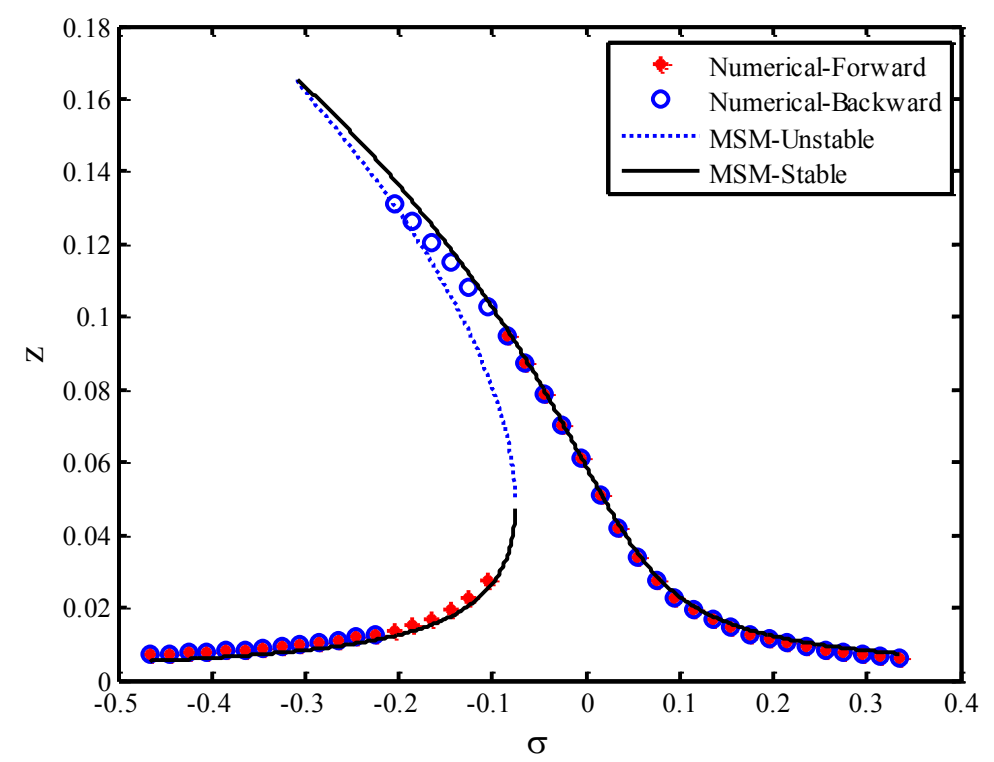

Figure 2: Frequency response curve of the arch resonator obtained by numerical simulation of equation (12) and the given MSM analytical frequency response (28) for $a_{1}=7.993, h=0.34$, $b=128, R=0.01$ and $m=0.03$

\section{Chaos prediction}

In this section we aim to use various analytical approaches to predict the possibility of chaos in parameter space of the arch resonator. Inspecting various categories given in Table 1, one can find that category A does not show chaotic behavior since snap-through is not possible in this category. Also, category $\mathrm{C}$ lacks the possibility of chaos because the pull-in instability eventuates in the structure collapse, immediately after the precipitate snap-through motion. Thus, only the arch resonators represented by the category B are vulnerable to chaos. Provided that the arch initial rise parameter $h$ and the DC voltage parameter $b$ impose the static bistability of the arch, represented by category B in Table 1, we can investigate the other effective parameters including $R, s$ and $m$ to derive dynamic requirements for the chaotic vibrations.

We can implement analytical tools to investigate the possibility of chaos in the arch's parameter space. To this aim, two different methods including the Melnikov and the multi-well potential criteria [34,35,38], will be employed to determine the necessary conditions for the chaotic behavior. To use the multi-well potential for chaos prediction, we will implement the obtained MSM-based frequency response equation. Details of these two criteria will be described in the following subsections. 


\subsection{Melnikov's Method}

In order to apply the Melnikov's method, the governing equation (12) is rewritten in the state space form using new variables $q_{1}=q$ and $q_{2}=q$ :

$$
\begin{aligned}
& q_{1} \&=q_{2} \\
& q \underset{2}{\&}=-\left(1+2 a h^{2}\right) q_{1}+3 a h q_{1}^{2}-a q_{1}^{3}+\frac{b}{2 b_{11} \sqrt{\left(1+h-q_{1}\right)^{3}}}+e\left(-\hat{m} q_{2}+\frac{\hat{R} b \cos \left(w_{0} t\right)}{b_{11} \sqrt{\left(1+h-q_{1}\right)^{3}}}\right)
\end{aligned}
$$

The Melnikov's relation is a function of distance between stable and unstable manifolds of the corresponding homoclinic orbits [32,39]:

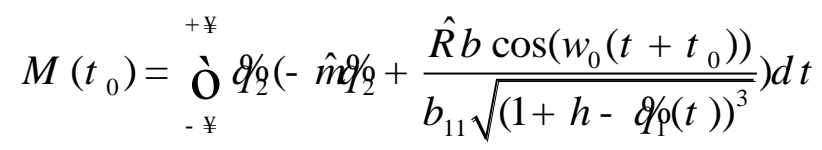

where, $q=\left(q_{p}, q_{q}\right)$ is the solution to unperturbed and undamped homoclinic orbit and is given by the following equation:

$$
q_{q}=\frac{d q_{p}}{d t}= \pm \sqrt{2\left[U\left(q_{s}\right)-U\left(q_{p}\right)\right]}
$$

where $q_{s}$ is the snap-through saddle point. Since there is no closed form solution to the unperturbed homoclinic orbit of equation (31), we approximate it by the homoclinic orbit of the well-known Duffing equation [32]. To this aim, the snap-through saddle point $q_{s}$ and its left point $q_{e l}$, with the property $U\left(q_{e l}\right)=U\left(q_{s}\right)$, are found. These values are then introduced to the following equations of Duffing homoclinic orbit [40]:

$q \rho(t)=-q_{e l} \operatorname{sech}(\sqrt{q} t)+q_{s}$

$q_{q}(t)=q_{e l} \sqrt{q} \operatorname{sech}(\sqrt{q} t)^{\prime} \tanh (\sqrt{q} t)$

where $q=-m_{1}$. Note that $q$ is positive since $m_{1}$ is obtained using unstable saddle point $q_{s}$ as the equilibrium point. Provided that $q_{e l}$ in the above equations is replaced by $q_{e r}$ with the property $U\left(q_{e r}\right)=U\left(q_{s}\right)$ and $q_{e r}>q_{s}$, the right half of the homoclinic orbit is approximated by equations (32) and (33). Figure 3 compares the homoclinic orbits approximated by equations (32) and (33) with those obtained by numerical simulation of unforced and unperturbed equation of motion with the initial conditions at the saddle point, for $h=0.35$ and two values of $b=134$ and $b=126$. It is clear that the approximation is accurate for the near-symmetric homoclinic orbits; while, the approximation accuracy weakens at the extremums as the homoclinic orbit becomes more asymmetric. Nevertheless, we will show that the obtained Melnikov criterion is valid for a wide range of parameters. 


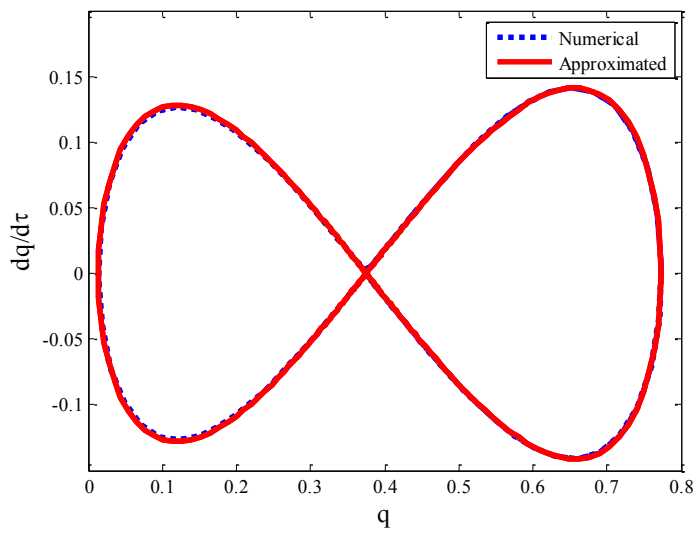

(a)

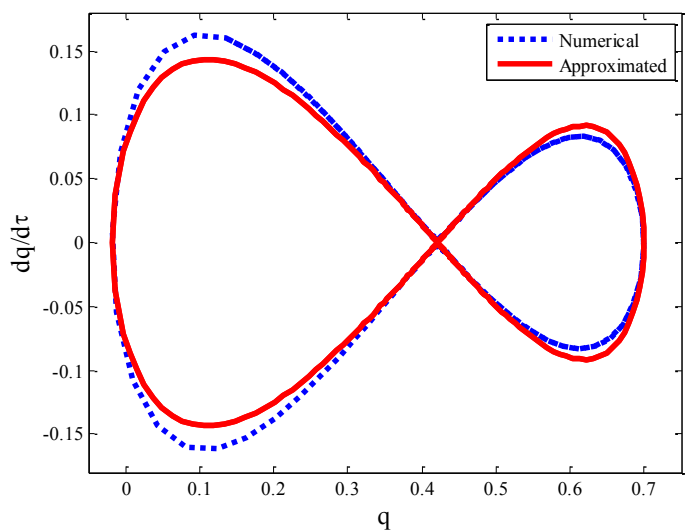

(b)

Figure 3: Approximated and numerical homoclinic orbits for $h=0.35$ with $b=134$ (a) and

$$
b=126(\mathrm{~b})
$$

However, using the approximated homoclinic orbit, Melnikov's function is rewritten in the following form:

$M\left(t_{0}\right)=\hat{m} I_{1}+\hat{R} I_{2}$

where the integrals $I_{1}$ and $I_{2}$ are given by the following relations:

$$
\begin{aligned}
& I_{1}=\stackrel{+¥}{\stackrel{+}{\mathbf{O}}}-q q_{2}^{2} d t \\
& I_{2}=\underset{-¥}{+¥} \frac{b q_{Q}(t) \cos \left(w_{0}\left(t+t_{0}\right)\right)}{b_{11} \sqrt{\left(1+h-q_{q}(t)\right)^{3}}} d t
\end{aligned}
$$

The first integral can be obtained analytically by symbolic calculations using Mathematica [41]. In order to obtain the second integral, we can expand the nonlinear term in its denominator up to the second order using Taylor's expansion method. Then, the resulting two integrals are solved symbolically using Mathematica. This yields the following solutions:

$I_{1}=-\frac{2}{3} \sqrt{q} q_{e}^{2}$

$I_{2}=\left[\frac{-p q_{e} b w_{0}}{b_{11} \sqrt{q(1+h)^{3}}} \operatorname{sech}(w \varnothing)+\frac{3 b p q_{e} w_{0}}{4 b_{11} q \sqrt{(1+h)^{5}}}\left(q_{e} w_{0} \operatorname{cosech}(w \varnothing)-2 \sqrt{q} q_{s} \operatorname{sech}(w \varnothing)\right)\right] \sin \left(w_{0} t_{0}\right)$

where $w \phi=p w_{0} /(2 \sqrt{q})$. Figure 4 compares the approximated Melnikov function using equations (37) and (38) with its actual value obtained by numerical evaluation of equations (35) and (36) for typical values of parameters. One can find that the relative error does not exceed $4.6 \%$ for the given parameter values, and this justifies construction of the Melnikov function using analytical solution given by equations (37) and (38). 


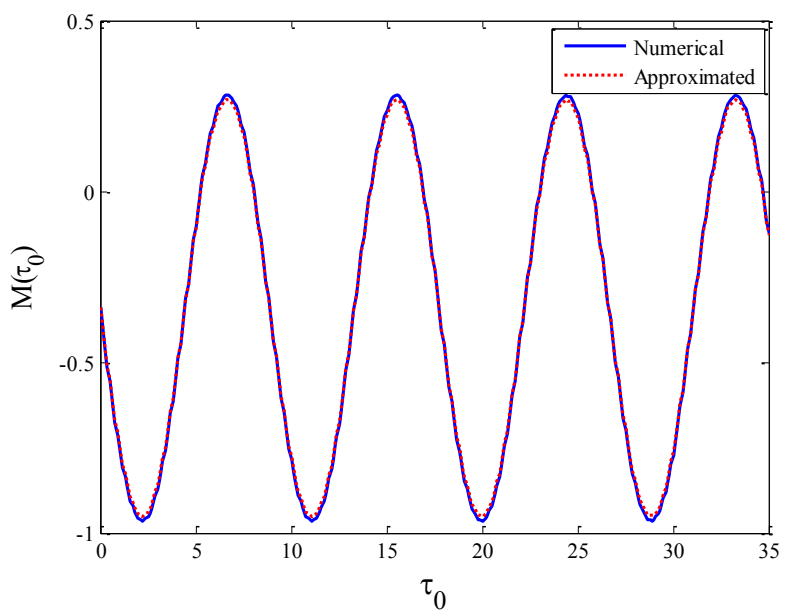

Figure 4: Numerical and approximated Melnikov function for $h=0.32, b=122, m=0.1$,

$$
R=0.04 \text { and } s=-0.1
$$

Provided that $M\left(t_{0}\right)=0$ is satisfied for some $t_{0}$, Melnikov's method predicts possibility of chaotic vibrations [39]. Based on this condition, we are left with the following inequality as a necessary but not sufficient condition for the chaotic vibrations:

$$
R_{c M E L}^{3} m\left|\frac{8 b_{11} q_{e}^{2} \sqrt{q^{3}(1+h)^{5}}}{p b q_{e} w_{0}\left[-12 \sqrt{q}(1+h) \operatorname{sech}(w \phi)+9 q_{e} w_{0} \operatorname{cosech}(w \varnothing)-18 q_{e} \sqrt{q} \operatorname{sech}(w \varnothing)\right]}\right|
$$

where $q_{e}$ can be replaced either with $q_{e l}$ or with $q_{e r}$ depending on the side of the homoclinic orbit for which we calculate the inequality. Further discussion on this will be presented in the following sections. Since the Melnikov's criterion is conservative in practice, we may expand the nonlinear term in equation (36) only up to the first order. This neglects second and third terms in the right hand of equation (38) and leaves us with a simpler but less precise inequality as the Melnikov's criterion:

$$
R_{c M E L}^{3} m\left|\frac{2 b_{11} q q_{e} \sqrt{(1+h)^{3}}}{3 p b w_{0} \operatorname{sech}(w \varnothing)}\right|
$$

For a specific arch with known initial rise parameter $h$ and known DC voltage parameter $b$ the quasi-static behavior is determined; and the arch is classified in the category B of Table 2 for certain values of $h$ and $b$. Thus, for such an arch, equation (39) gives the critical value of the harmonic excitation parameter $R$, below which chaos does not happen, as a function of the harmonic excitation frequency and the damping coefficient (see Figure 5a). However, equation (39), or equation (40) as its less precise counterpart, provides a rapid tool for prediction of chaos in the investigated arch resonator, and enables rapid investigation of the effects of various parameters. For instance, one can clearly detect from the Melnikov criterion given by equations 
(39) or (40) that the critical value of $R$ for initiation of chaos increases linearly with the damping coefficient. Effects of the other parameters can also be scrutinized rapidly. This is the most important functionality of the analytical tools for chaos prediction. In the next subsection, another analytical tool for chaos prediction is presented.

\subsection{The Maximum Velocity Criterion (MVC)}

Melnikov's method predicts any intersection of the system trajectory in its phase plane with the snap-through homoclinic orbit. Thus, this criterion is suitable for prediction of transient chaotic vibrations [32]. Moreover it is too conservative and seems to be ineffective in the prediction of steady state chaotic vibrations. In order to obtain a more effective criterion for prediction of chaotic vibrations we refer to the analytical solution for the frequency response of the arch resonator obtained with the MSM and given by equation (28). Using this analytical relation and based on a previously developed criterion for chaos prediction, we can derive an appropriate tool for chaos detection in the investigated bistable MEMS resonator.

As described by Nejat Pishkenari et al. [34] and Moon [38], chaotic vibrations are expected provided that the maximum velocity of the system exceeds the maximum velocity of the corresponding homoclinic orbit which occurs at the local minima of the potential energy function. Thus, one can easily calculate the maximum velocity of the homoclinic orbit, by equating the Hamiltonian of the system at the saddle point and the equilibrium point:

$V_{m h}=\sqrt{2\left[\mathrm{U}\left(\mathrm{q}_{s}\right)-\mathrm{U}\left(\mathrm{q}_{0}\right)\right]}$

where $V_{m h}$ represents the maximum velocity of the homoclinic orbit, while $q_{s}$ is the saddle point and $q_{0}$ is either of the equilibrium points. Along this line, the corresponding amplitude of vibration is obtained:

$$
z_{C}=\frac{V_{m h}}{w_{0}}
$$

Substituting $z$ in equation (28) with the critical value given by equation (42) and solving the resulted equation for the critical excitation $R_{c}$, we can derive the minimum required excitation for initiation of the chaotic vibrations:

$$
R_{c}=\frac{V_{m h} l \sqrt{\left(4 l V^{2} g-32 s l^{2} w_{0}^{2}\right)^{2}+m^{2}\left(V^{2} g-(4 l+m)^{2} w_{0}^{2}\right)^{2}}}{\left|s_{0}\right|\left(16 l^{2}+m^{2}\right) w_{0}^{3}}
$$

For the known values $h$ and $b$ all parameters in equation (43) are known except $m$ and $w_{0}$. Thus, one can obtain the critical excitation amplitude $R_{c}$ as a function of excitation frequency $w_{0}$ (or equivalently $s$ ) and the damping parameters $m$. Figure 5 displays contour of the predicted critical value of $R_{c}$ as a function of both $m$ and $s$ for an arch of $h=0.30$ and 
$b=117$. Figure 5a displays the functionality of $R_{c}$ given by equation (39) as the Melnikov criterion; and the same is done in Figure 5b using equation (43) as the MVC-MSM result. One can find that, regarding the bistable nature of the arch, either of equations (39) or (43) are possible to be evaluated using the parameters calculated for the left or the right sides of the homoclinic orbit. Thus, each formula results in two separate critical values of $R$. In Figure 5, both criteria are calculated for the left side of the corresponding homoclinic orbit.

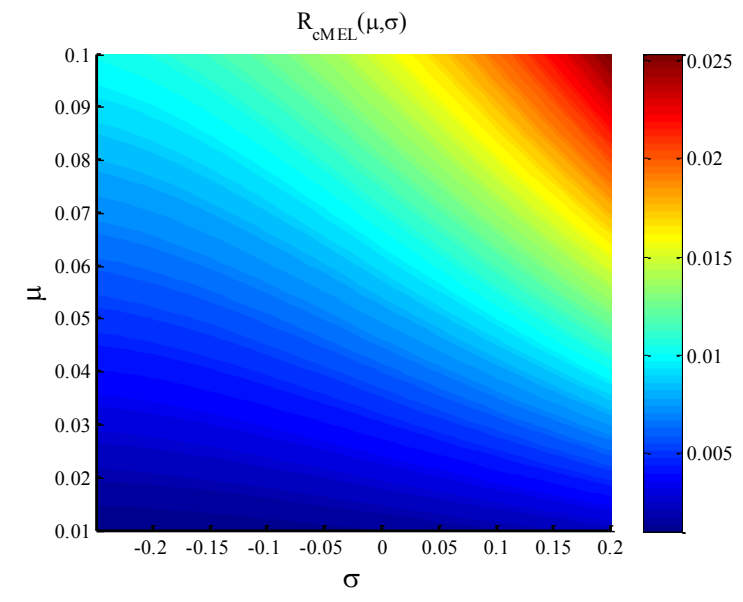

(a)

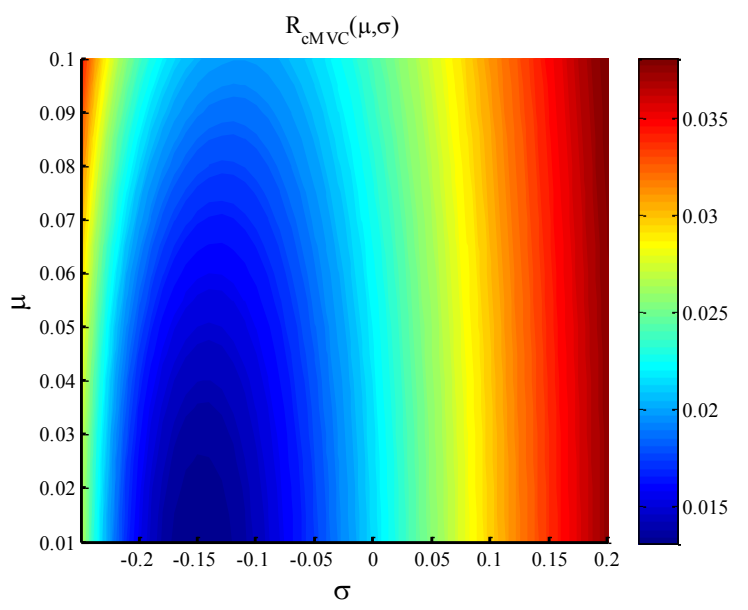

(b)

Figure 5: Contour of the critical value of excitation amplitude below which chaos is not expected as a function of excitation frequency and damping parameter for $h=0.30$ and $b=117$; a) the Melnikov criterion, b) the MVC-MSM criterion

Figure 5 shows that both criteria predict a positive slope with respect to the damping coefficient, suggesting that the maximum value of $R$ which can be tolerated before undergoing chaos increases as the damping coefficient grows. Also, the Melnikov criterion presents higher rate of growth of $R$ as a function of $m$. Furthermore, the functionality is the same for $s$ as predicted by the Melnikov method. However, the MVC-MSM criterion shows a minimum value of $R_{c}$ for the negative value of $s$. This is consistent with the softening type frequency response associated with the parameters of the arch used in Figure 5, which suggests that the maximum amplitude of the response occurs at negative values of $s$. Provided that the hardening type behavior arises due to the geometrical and actuation parameters, critical value of $R$ for chaos is expected to take place in the positive values of $s$. Therefore, the situation for chaos must be more critical at such values of excitation frequency. As the actuation frequency further increases, the amplitude of the system response decreases (see Figure 2); thus, the MVC-MSM criterion predicts higher safe value for the harmonic excitation amplitude $R$. These observations should be validated by appropriate numerical simulations; this is done in the next section. 


\section{Numerical Simulations}

In this section, we have investigated the chaotic vibrations of the system, and have validated the obtained analytical criteria, based on the numerical simulation of equation (12). To this aim, several couples of $h$ and $b$ are selected within the bistability region represented by category B in Table 2. Then, for each couple, several distinct values for $m$ representing resonators with various quality factors are assigned. Then for each set of $h, b$ and $m$, six separate values for the actuation frequency near the natural frequency of the arch are selected. Using the selected parameters, we solve equation (12) by an adaptive Runge-Kutta method with a maximum timestep equal to $1 \%$ of the forcing load period. Numerical simulations start with the system initially at rest in the equilibrium state with lower potential energy. To study the effects of actuation amplitude, simulations beginning with small values of $R$ are performed for a long time, and after recording the corresponding Poincare section by sampling the system trajectory with actuation frequency, $R$ is increased for a small value $d R=0.001$ and the procedure is continued until the simulation is halted due to the dynamic pull-in. At each step, the final states of the system at the end of the integration are chosen as the initial condition for the integration in the next step. Bifurcation diagrams versus $R$ are obtained for each set of parameters $h, b, m$ and $s$.

Figure 6 presents a typical bifurcation diagram, showing the $q$ component of the Poincare section versus the excitation amplitude parameter $R$. As shown in this bifurcation diagram, the system presents periodic vibrations for very small excitation amplitudes. As the AC actuation amplitude increases, the system trajectory approaches the snap-through homoclinic orbit; thus, the system undergoes homoclinic chaos. The first critical value of $R$ which results in the chaotic vibration is found as $R_{c}=0.023$ from Figure 6. Lyapunov exponents and the associated Poincare section with this critical value are shown in Figure 7, confirming the chaotic nature of the vibrations, due to the positivity of the maximum Lyapunov exponent in Figure $7 \mathrm{a}$ and formation of a strange attractor in Figure $7 \mathrm{~b}[39,42]$. The Melnikov criterion given by equation (39) predicts critical value of $R_{c}=0.007$ and $R_{c}=0.020$ using the left and right sides of the corresponding homoclinic orbit, respectively. Also, the MVC-MSM criterion given by equation (43) predicts critical values of $R_{c}=0.009$ and $R_{c}=0.025$ when performing calculations for the left and right sides of the corresponding homoclinic orbit, respectively. 


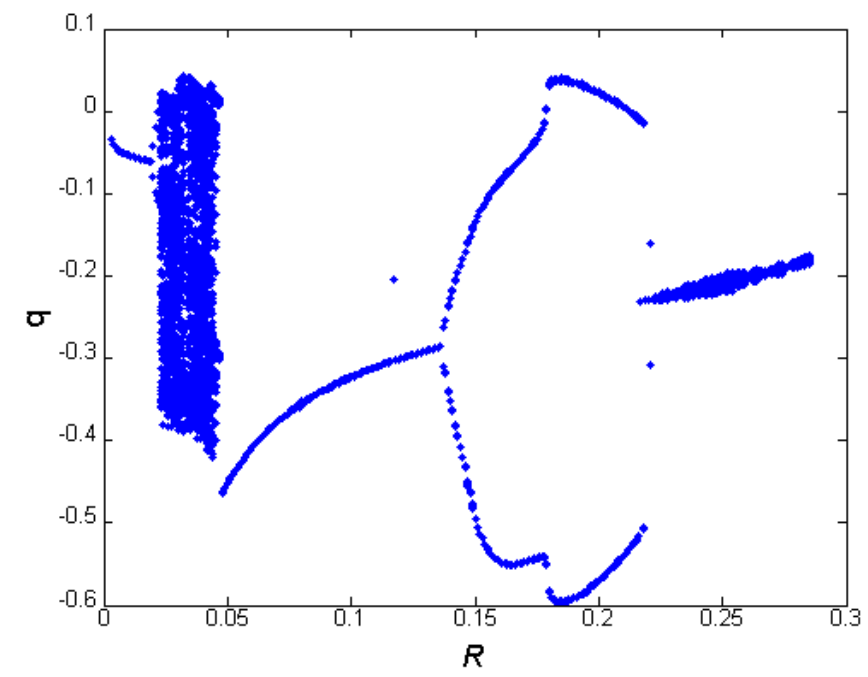

Figure 6: Bifurcation diagram obtained by numerical simulation of the arch resonator with

$$
h=0.30, b=120, \quad m=0.1 \text { and } w_{0}=0.47
$$

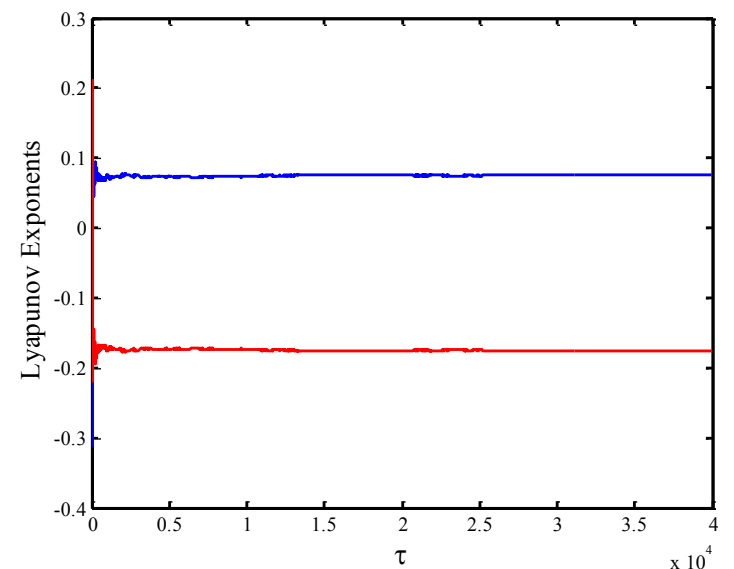

(a)

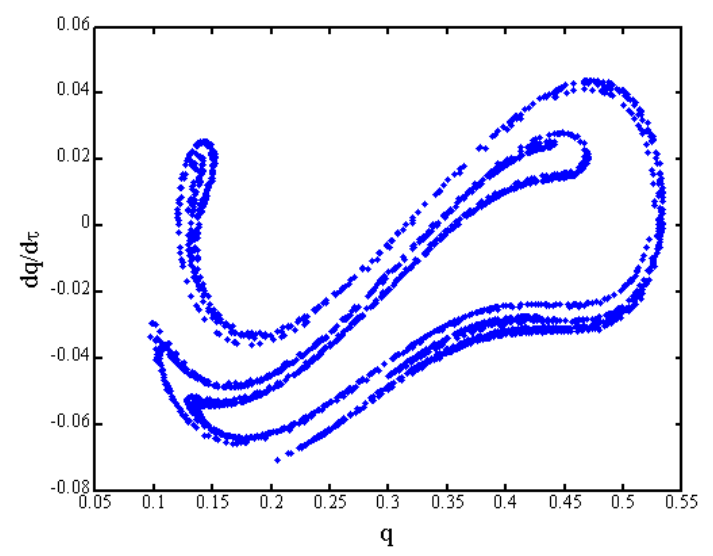

(b)

Figure 7: Lyapunov exponents (a) and the corresponding Poincare section (b) for parameter values associated with Figure 6 with $R=0.023$

For better evaluation of the capability of the obtained analytical tools in prediction of the first critical value of $R$ which results in chaos, we can plot the critical values obtained from numerical bifurcation diagrams together with the analytical predictions. Figure 8 illustrates the critical values of $R$ obtained by application of the Melnikov method, equation (39), and the MVC-MSM criterion, equation (43), to the left and right sides of the corresponding homoclinic orbit. Numerically obtained critical values of $R$ are also shown in Figure 8 confirming a good agreement of the MVC-MSM criterion with the numerical simulations. As expected, Figure 8 confirms that calculation of the analytical formula with the parameters associated with either side 
of the homoclinic orbit yields different values of $R_{c}$. Comparing Figure 8a having a nearsymmetric homoclinic orbit with Figure $8 \mathrm{~b}$ showing an asymmetric homoclinic orbit, we can find that for obtaining the critical value by the analytical formula one should calculate the formula for both sides of the homoclinic orbit and select the smaller amount as the critical value. The same approach should be used for application of the Melnikov method. Furthermore, using equation (44) for calculation of the average relative error $\bar{E}$ with $N=6$ denoting the number of available numerical data, we find that the average relative error equals to $13 \%$ and $87 \%$ for the best predictions given by the MVC-MSM and the Melnikov criteria associated with Figure 8a, respectively. Also, for Figure 8 b, values of $\bar{E}$ are found to be $16 \%$ and $82 \%$ for the MVC-MSM and the Melnikov criteria, respectively. Thus, Figure 8 confirms that the Melnikov method presents a rather conservative criterion for steady state chaos prediction at least for the small damping values for which Figure 8 is obtained. The relation for the average relative error is given by:

$$
\bar{E}=\frac{1}{N} \underset{j=1}{N} \frac{\left.\left|R_{c-\text { Analytical }}\left(m, w_{0}(\mathrm{j})\right)-R_{c-\text { Numerical }}\right|\right|^{\prime} 100}{R_{c-\text { Numerical }}}
$$

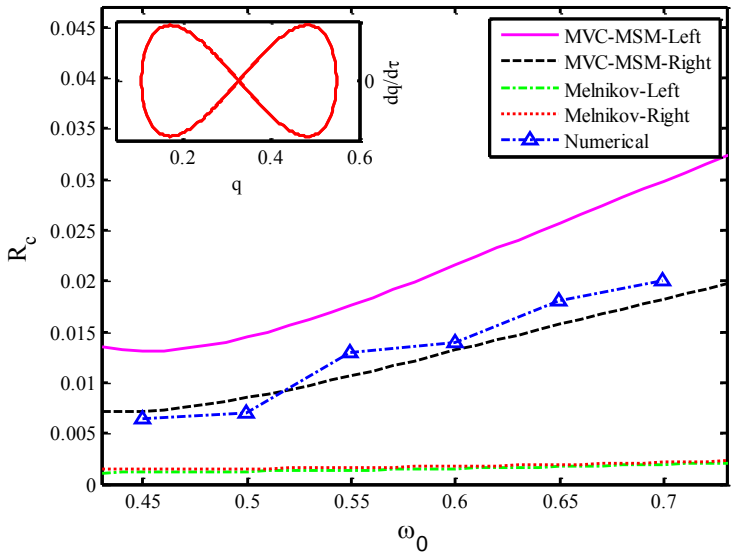

(a)

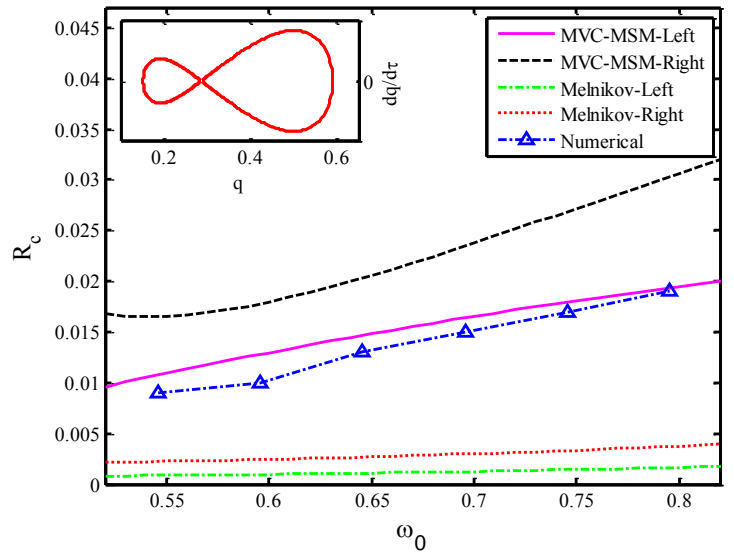

(b)

Figure 8: The first critical value of $R$ resulting in the chaotic vibrations versus the actuation frequency obtained by numerical simulations together with the MVC-MSM and the Melnikov criteria calculated for either sides of the homoclinic orbit for parameter values of $h=0.30$, $m=0.01$ and a) $b=117$; b) $b=120$. Insets display the corresponding homoclinic orbit

As shown in Figure 5a, the Menikov method is more sensitive to variations in the damping ratio. Thus, for better study of the validation of the analytical formula at various damping parameters, we need to study the critical values of $R$ versus the damping parameter. Figure 9 depicts the numerically obtained critical values of $R$ versus the damping parameter together with the corresponding values given by the Melnikov method and the MVC-MSM criterion for the left 
and right sides of the homoclinic orbit. Figure 9a is plotted for a fixed value of actuation frequency $w_{0}=0.8512$ near the natural frequency of the arch and a fixed value of DC voltage parameter $b=120$ resulting in an asymmetric homoclinic orbit. The same is done in Figure $9 \mathrm{~b}$ with $w_{0}=0.7577$ and $b=122.5$ resulting in a near-symmetric homoclinic orbit. Figure 9 shows that the MVC-MSM criterion is less sensitive to variations in the damping parameter of the resonator. However, the minimum value of the MVC-MSM criterion for left and right sides of the homoclinic orbit better predicts chaos for smaller values of damping coefficient. This is consistent with the assumption with which equation (28) is derived; i. e. a resonator with high quality factor (low damping ratio). As the damping ratio increases, accuracy of the response amplitude predicted by equation (28) decreases; and consequently, the chaos prediction capability weakens. Furthermore, Figure 9 shows that the chaos prediction given by the Melnikov method, equation (39), is more sensitive to changes in damping parameter; nevertheless, this criterion results in a conservative prediction, as expected. Additionally, the average relative error for the best predictions associated with Figure 9a and given by the MVCMSM and the Melnikov criteria are found to be $34 \%$ and 51\%, respectively; while, for Figure 9b, these error values are equal to $23 \%$ and $53 \%$ for the MVC-MSM and the Melnikov criteria, respectively.

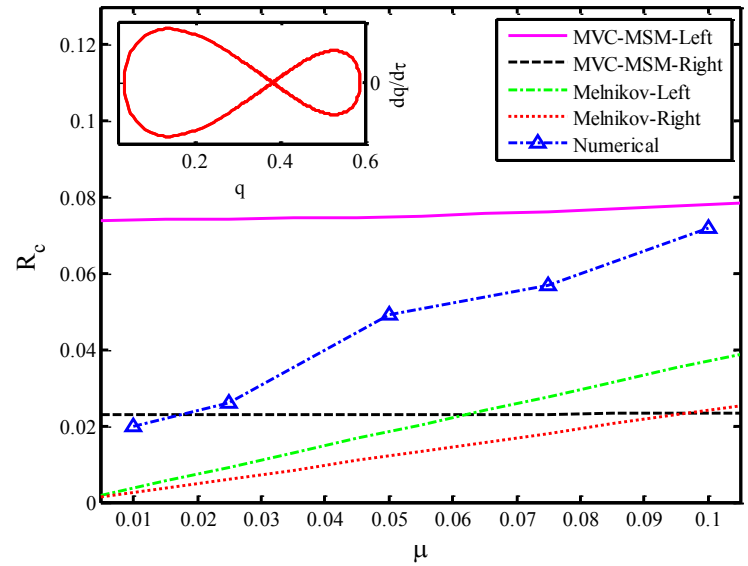

(a)

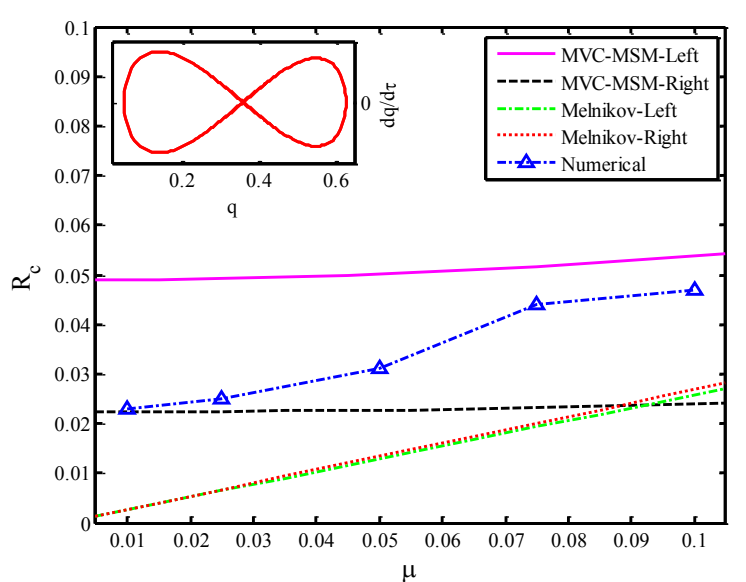

(b)

Figure 9: The first critical value of $R$ resulting in the chaotic vibration versus the damping parameter obtained by numerical simulations together with the MVC-MSM and the Melnikov criteria calculated for either sides of the homoclinic orbit for parameter values of $h=0.32$, and a) $b=120, w_{0}=0.8512$; b) $b=122.5, w_{0}=0.7577$. Insets display the corresponding homoclinic orbits.

\section{Summary and Conclusions}

Investigation of the nonlinear dynamics and chaos in bistable micro/nano resonators using analytical approaches is the main scope of the present research. To this aim, we have studied the 
chaotic behavior of an electrostatically actuated double clamped shallow arch micro resonator. The arch is simultaneously loaded by static DC voltage, which determines the static stability nature of the arch together with its geometrical parameters, and by harmonic AC actuation, with a frequency close to the first fundamental frequency, which triggers resonant vibrations of the arch. A reduced order single degree of freedom model, obtained by the application of the single mode Galerkin decomposition method to the governing Euler-Bernoulli shallow arch equation with mid-plane stretching effect, have been used for the investigations.

Based on the shape of the non-dimensional potential energy, studied as a function of the DC voltage parameter and arch's initial curvature, we have classified the possible arch configurations into three main categories; one of which presents both bistability and capability of dynamic snap-through motion without pull-in instability. Chaos is possible to occur in the forced vibrations of this category possessing a double-well potential function which results in the constitution of a mainly asymmetric homoclinic orbit in the corresponding phase plane of the resonator. Thus, homoclinic chaos can be expected for certain values parameters in this category.

We have developed analytical solutions to predict homoclinic chaos in the parameter space of the investigated arch resonator. Approximating the arch's homoclinic orbit by that of the wellknown Duffing equation, we have applied the Melnikov's method and have derived an analytical necessary-but-not-sufficient condition for prediction of the smallest critical value of the excitation amplitude parameter which eventuates in the chaotic vibrations. Moreover, we have implemented a heuristic criterion developed for prediction of steady state chaos in multi-well potential systems. This method, the Maximum Velocity Criterion (MVC), predicts initiation of chaos provided that the maximum velocity of the system response approaches that of the corresponding homoclinic orbit. To be able to use this criterion, an analytical solution for the amplitude of the system response is needed. We have used such a solution, obtained by application of the Multiple Scales Method (MSM) to the arch's equation of motion, together with the maximum velocity criterion to derive an analytical solution for prediction of chaos in the parameter space of the resonator.

Numerical simulations are performed to validate the obtained analytical expressions. Bifurcation diagrams, given by the numerical simulations, describe the smallest critical amplitude parameter which results in the steady state chaotic vibrations. Using various bifurcation diagrams, we have derived the critical amplitude parameter versus the excitation frequency and the damping parameter. Comparing the numerical results with those predicted by the analytical expressions, we have validated the obtained expressions. Regarding the bistable nature of the arch, each analytical solution can be calculated with parameters associated with one of the stable configurations. Thus, for a bistable arch with asymmetric homoclinic orbit, each analytical expression yields two separate critical values; the minimum of which should be used for chaos prediction. Numerical results confirm that the Melnikov method presents a rather conservative criterion especially for the arch resonators with high quality factors. However, the MVC-MSM criterion provides better prediction for the chaos. As the damping coefficient increases, accuracy 
of the MSM solution decreases; thus, the given MVC-MSM criterion is more accurate for arches with relatively high quality factors.

Regarding the rapid development of bistable arch resonators in various applications such as bandpass filters, the results of this work will be useful for quick determination of chaotic bands in the parameter space of the arch. This can be used in the design procedure of the investigated family of the arch resonators for avoiding chaos in the resonator's response. Moreover, a similar approach for the analysis and prediction of chaos can be pursued in other engineering systems possessing multi-well potential and asymmetric homoclinic orbit. Experimental validation of the obtained analytical expressions for chaos prediction is required and is left as a future work.

\section{Acknowledgment}

The authors would like to thank the Iranian National Science Foundation (INSF) for their financial support.

\section{References}

[1] A. Uranga, J. Verd, E. Marigó, J. Giner, J.L. Muñóz-Gamarra, N. Barniol, Exploitation of non-linearities in CMOS-NEMS electrostatic resonators for mechanical memories, Sensors Actuators A Phys. 197 (2013) 88-95.

[2] H.M. Ouakad, An electrostatically actuated MEMS arch band-pass filter, Shock Vib. 20 (2013) 809-819. doi:10.3233/SAV-130786.

[3] Y.B. Wu, G.F. Ding, C.C. Zhang, J. Wang, S.P. Mao, H. Wang, Magnetostatic bistable MEMS switch with electrothermal actuators, Electron. Lett. 46 (2010) 1074-1075.

[4] X. Miao, X. Dai, P. Wang, G. Ding, X. Zhao, Design, fabrication and characterization of a bistable electromagnetic microrelay with large displacement, Microelectronics J. 42 (2011) 992-998.

[5] J. Sutanto, R. Luharuka, P.J. Hesketh, Y.H. Berthelot, Designing and fabricating electromagnetically actuated microvalves for MEMS applications, Sensors Mater. 19 (2007) 35-56.

[6] M.I. Younis, H.M. Ouakad, F.M. Alsaleem, R. Miles, W. Cui, Nonlinear Dynamics of MEMS Arches Under Harmonic Electrostatic Actuation, J. Microelectromechanical Syst. 19 (2010) 647-656.

[7] H.M. Ouakad, M.I. Younis, The dynamic behavior of MEMS arch resonators actuated electrically, Int. J. Non. Linear. Mech. 45 (2010) 704-713. 
[8] A. Michael, C.Y. Kwok, Design criteria for bi-stable behavior in a buckled multi-layered MEMS bridge, J. Micromechanics Microengineering. 16 (2006) 2034-2043.

doi:10.1088/0960-1317/16/10/016.

[9] J.S. Han, J.S. Ko, Y.T. Kim, B.M. Kwak, Parametric study and optimization of a microoptical switch with a laterally driven electromagnetic microactuator, J. Micromechanics Microengineering. 12 (2002) 939-947. doi:10.1088/0960-1317/12/6/326.

[10] M. Sulfridge, T. Saif, N. Miller, M. Meinhart, Nonlinear Dynamic Study of a Bistable MEMS : Model and Experiment, J. Microelectromechanical Syst. 13 (2004) 725-731.

[11] J. Qiu, J.H. Lang, A.H. Slocum, A Curved-Beam Bistable Mechanism, J. Microelectromechanical Syst. 13 (2004) 137-146.

[12] S. Krylov, B.R. Ilic, D. Schreiber, S. Seretensky, H. Craighead, The pull-in behavior of electrostatically actuated bistable microstructures, J. Micromechanics Microengineering. 18 (2008) 055026. doi:10.1088/0960-1317/18/5/055026.

[13] W.-M. Zhang, H. Yan, Z.-K. Peng, G. Meng, Electrostatic pull-in instability in MEMS/NEMS: A review, Sensors Actuators A Phys. 214 (2014) 187-218. doi:10.1016/j.sna.2014.04.025.

[14] G.M. Rebeiz, RF MEMS: Theory, Design and Technology, Wiley, New York, 2003.

[15] F. Lakrad, M. Belhaq, Suppression of pull-in in a microstructure actuated by mechanical shocks and electrostatic forces, Int. J. Non. Linear. Mech. 46 (2011) 407-414. doi:10.1016/j.ijnonlinmec.2010.10.006.

[16] H. Rokni, R.J. Seethaler, A.S. Milani, S. Hosseini-hashemi, X. Li, Analytical closed-form solutions for size-dependent static pull-in behavior in electrostatic micro-actuators via Fredholm integral equation, Sensors Actuators A. Phys. 190 (2013) 32-43. doi:10.1016/j.sna.2012.10.035.

[17] A.H. Nayfeh, M.I. Younis, E.M. Abdel-Rahman, Dynamic pull-in phenomenon in MEMS resonators, Nonlinear Dyn. 48 (2007) 153-163.

[18] S. Krylov, N. Dick, Dynamic stability of electrostatically actuated initially curved shallow micro beams, Contin. Mech. Thermodyn. 22 (2010) 445-468. doi:10.1007/s00161-0100149-6.

[19] K. Das, R.C. Batra, Pull-in and snap-through instabilities in transient deformations of microelectromechanical systems, J. Micromechanics Microengineering. 19 (2009) 035008. doi:10.1088/0960-1317/19/3/035008. 
[20] Y. Zhang, Y. Wang, Z. Li, Y. Huang, D. Li, Snap-Through and Pull-In Instabilities of an Arch-Shaped Beam Under an Electrostatic Loading, J. Microelectromechanical Syst. 16 (2007) 684-693.

[21] J. Casals-terré, A. Fargas-marques, A.M. Shkel, Snap-Action Bistable Micromechanisms Actuated by Nonlinear Resonance, J. Microelectromechanical Syst. 17 (2008) 1082-1093.

[22] H.M. Ouakad, M.I. Younis, On using the dynamic snap-through motion of MEMS initially curved microbeams for filtering applications, J. Sound Vib. 333 (2014) 555-568.

[23] F. Tajaddodianfar, H. Nejat Pishkenari, M. Hairi Yazdi, E. Maani Miandoab, On the Dynamics of Bistable Micro/Nano Resonators: Analytical Solution and Nonlinear Behavior, Commun. Nonlinear Sci. Numer. Simul. 20 (2015) 1078-1089. doi:10.1016/j.cnsns.2014.06.048.

[24] A. Seleim, S. Towfighian, E. Delande, E. Abdel-Rahman, G. Heppler, Dynamics of a close-loop controlled MEMS resonator, Nonlinear Dyn. 69 (2011) 615-633. doi:10.1007/s11071-011-0292-z.

[25] S.-H. Yin, B.I. Epureanu, Experimental Enhanced Nonlinear Dynamics and Identification of Attractor Morphing Modes for Damage Detection, J. Vib. Acoust. 129 (2007) 763. doi:10.1115/1.2775507.

[26] K. Park, Q. Chen, Y.-C. Lai, Energy enhancement and chaos control in microelectromechanical systems, Phys. Rev. E. 77 (2008) 026210. doi:10.1103/PhysRevE.77.026210.

[27] F.N. Mayoof, M. a. Hawwa, Chaotic behavior of a curved carbon nanotube under harmonic excitation, Chaos, Solitons \& Fractals. 42 (2009) 1860-1867. doi:10.1016/j.chaos.2009.03.104.

[28] M.P. Aghababa, Chaos in a fractional-order micro-electro-mechanical resonator and its suppression, Chinese Phys. B. 21 (2012) 100505. doi:10.1088/1674-1056/21/10/100505.

[29] T.D. Amorim, W.G. Dantas, A. Gusso, Analysis of the chaotic regime of MEMS/NEMS fixed-fixed beam resonators using an improved 1DOF model, Nonlinear Dyn. (2014). doi:10.1007/s11071-014-1715-4.

[30] M.S. Siewe, U.H. Hegazy, Homoclinic bifurcation and chaos control in MEMS resonators, Appl. Math. Model. 35 (2011) 5533-5552. doi:10.1016/j.apm.2011.05.021.

[31] B.E. Demartini, H.E. Butterfield, J. Moehlis, K.L. Turner, Chaos for a Microelectromechanical Oscillator Governed by the Nonlinear Mathieu Equation, J. Microelectromechanical Syst. 16 (2007) 1314-1323. 
[32] H.S. Haghighi, A.H.D. Markazi, Chaos prediction and control in MEMS resonators, Commun. Nonlinear Sci. Numer. Simul. 15 (2010) 3091-3099.

doi:10.1016/j.cnsns.2009.10.002.

[33] a. H. Nayfeh, H.M. Ouakad, F. Najar, S. Choura, E.M. Abdel-Rahman, Nonlinear dynamics of a resonant gas sensor, Nonlinear Dyn. 59 (2010) 607-618.

doi:10.1007/s11071-009-9567-z.

[34] H.N. Pishkenari, M. Behzad, A. Meghdari, Nonlinear dynamic analysis of atomic force microscopy under deterministic and random excitation, Chaos, Solitons \& Fractals. 37 (2008) 748-762. doi:10.1016/j.chaos.2006.09.079.

[35] E. Maani Miandoab, H. Nejat Pishkenari, A. Yousefi-Koma, F. Tajaddodianfar, Chaos prediction in MEMS-NEMS resonators, Int. J. Eng. Sci. 82 (2014) 74-83. doi:10.1016/j.ijengsci.2014.05.007.

[36] E. Maani Miandoab, A. Yousefi-Koma, H. Nejat Pishkenari, F. Tajaddodianfar, Study of Nonlinear Dynamics and Chaos in MEMS/NEMS Resonators, Commun. Nonlinear Sci. Numer. Simul. (2014). doi:10.1016/j.cnsns.2014.07.007.

[37] E.M. Miandoab, A. Yousefi-Koma, H.N. Pishkenari, M. Fathi, Nano-resonator frequency response based on strain gradient theory, J. Phys. D. Appl. Phys. 47 (2014) 365303. doi:10.1088/0022-3727/47/36/365303.

[38] F.C. Moon, Chaotic Vibrations: An Introduction for Applied Scientists and Engineers, John Wiley \& Sons, 2004.

[39] S. Wiggins, Introduction to Applied Nonlinear Dynamical Systems and Chaos, Springer, 2003.

[40] J. Guckenheimer, P. Holmes, Nonlinear oscillations, dynamical systems, and bifurcations of vector fields, Springer-Verlag, New York, 1983.

[41] Wolfram Research, <www.wolfram.com>. (n.d.).

[42] A. Wolf, J.B. Swift, H.L. Swinney, J.A. Vastano, Determining Lyapunov Exponents from a Time Series, Phys. D. 16 (1985) 285-317. 